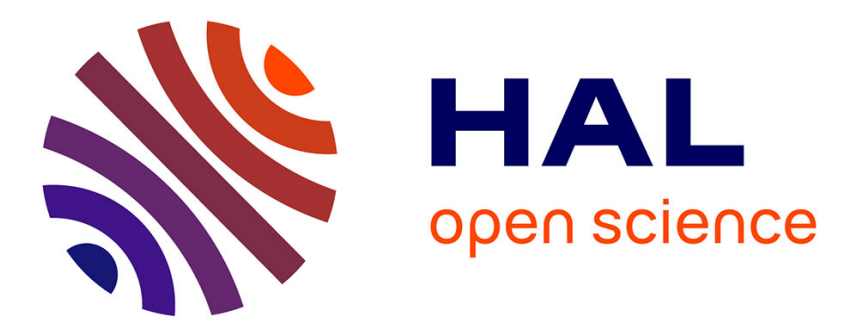

\title{
Weak Well-Posedness of Multidimensional Stable Driven SDEs in the Critical Case
}

\author{
Paul-Eric Chaudru de Raynal, Stephane Menozzi, Enrico Priola
}

\section{To cite this version:}

Paul-Eric Chaudru de Raynal, Stephane Menozzi, Enrico Priola. Weak Well-Posedness of Multidimensional Stable Driven SDEs in the Critical Case. Stochastics and Dynamics, 2020, 20 (6), pp.2040004. 10.1142/S0219493720400043 . hal-02434363

\section{HAL Id: hal-02434363 \\ https://hal.science/hal-02434363}

Submitted on 10 Jan 2020

HAL is a multi-disciplinary open access archive for the deposit and dissemination of scientific research documents, whether they are published or not. The documents may come from teaching and research institutions in France or abroad, or from public or private research centers.
L'archive ouverte pluridisciplinaire HAL, est destinée au dépôt et à la diffusion de documents scientifiques de niveau recherche, publiés ou non, émanant des établissements d'enseignement et de recherche français ou étrangers, des laboratoires publics ou privés. 


\title{
WEAK WELL-POSEDNESS OF MULTIDIMENSIONAL STABLE DRIVEN SDES IN THE CRITICAL CASE
}

\author{
PAUL-ÉRIC CHAUDRU DE RAYNAL, STÉPHANE MENOZZI, AND ENRICO PRIOLA
}

\begin{abstract}
We establish weak well-posedness for critical symmetric stable driven SDEs in $\mathbb{R}^{d}$ with additive noise $Z, d \geq 1$. Namely, we study the case where the stable index of the driving process $Z$ is $\alpha=1$ which exactly corresponds to the order of the drift term having the coefficient $b$ which is continuous and bounded. In particular, we cover the cylindrical case when $Z_{t}=\left(Z_{t}^{1}, \ldots, Z_{t}^{d}\right)$ and $Z^{1}, \ldots, Z^{d}$ are independent one dimensional Cauchy processes. Our approach relies on $L^{p}$-estimates for stable operators and uses perturbative arguments.
\end{abstract}

Keywords: Stable driven SDEs; Critical case; Martingale problem.

AMS Subject Classification: 60H10, 60H30, 35K65

\section{Statement of the Problem and main Results}

We are interested in proving well-posedness for the martingale problem associated with the following SDE:

$$
X_{t}=x+\int_{0}^{t} b\left(X_{s}\right) d s+Z_{t},
$$

where $\left(Z_{s}\right)_{s \geq 0}$ stands for a symmetric $d$-dimensional stable process of order $\alpha=1$ defined on some filtered probability space $\left(\Omega, \mathcal{F},\left(\mathcal{F}_{t}\right)_{t \geq 0}, \mathbb{P}\right)$ (cf. [2] and the references therein) under the sole assumptions of continuity and boundedness on the vector valued coefficient $b$ :

$$
\text { (C) The drift } b: \mathbb{R}^{d} \rightarrow \mathbb{R}^{d} \text { is continuous and bounded } 1
$$

Above, the generator $L$ of $Z$ writes:

$$
\begin{aligned}
L \varphi(x) & =\text { p.v. } \int_{\mathbb{R}^{d} \backslash\{0\}}[\varphi(x+z)-\varphi(x)] \nu(d z), \quad x \in \mathbb{R}^{d}, \quad \varphi \in C_{b}^{2}\left(\mathbb{R}^{d}\right), \\
\nu(d z) & =\frac{d \rho}{\rho^{2}} \tilde{\mu}(d \theta), \quad z=\rho \theta,(\rho, \theta) \in \mathbb{R}_{+}^{*} \times \mathbb{S}^{d-1} .
\end{aligned}
$$

(here $\langle\cdot, \cdot\rangle$ (or $\cdot$ ) and $|\cdot|$ denote respectively the inner product and the norm in $\mathbb{R}^{d}$ ). In the above equation, $\nu$ is the Lévy intensity measure of $Z, \mathbb{S}^{d-1}$ is the unit sphere of $\mathbb{R}^{d}$ and $\tilde{\mu}$ is a spherical measure on $\mathbb{S}^{d-1}$. It is well know, see e.g. 20] that the Lévy exponent $\Phi$ of $Z$ writes as:

$$
\Phi(\lambda)=\mathbb{E}\left[\exp \left(i\left\langle\lambda, Z_{1}\right\rangle\right)\right]=\exp \left(-\int_{\mathbb{S}^{d-1}}|\langle\lambda, \theta\rangle| \mu(d \theta)\right), \quad \lambda \in \mathbb{R}^{d},
$$

where $\mu=c_{1} \tilde{\mu}$, for a positive constant $c_{1}$, is the so-called spectral measure of $Z$. We will assume some non-degeneracy conditions on $\mu$. Namely we introduce assumption

(ND) There exists $\kappa \geq 1$ s.t.

$$
\forall \lambda \in \mathbb{R}^{d}, \kappa^{-1}|\lambda| \leq \int_{\mathbb{S}^{d-1}}|\langle\lambda, \theta\rangle| \mu(d \theta) \leq \kappa|\lambda|
$$

\footnotetext{
${ }^{1}$ The boundedness of $b$ is here assumed for technical simplicity. Our methodology could apply, up to suitable localization arguments, to a drift $b$ having linear growth.
} 
Notably, no regularity on the spectral measure itself is assumed. In particular, we do not assume that $\mu$ has a density with respect to the Lebesgue measure on $\mathbb{S}^{d-1}$. When such density exists and it is constant, we get, up to multiplicative factor, the usual fractional Laplacian $L=\Delta^{\frac{1}{2}}$. On the other hand, we can also consider the singular measure $\mu=\sum_{i=1}^{d} \frac{1}{2}\left(\delta_{e_{i}}+\delta_{-e_{i}}\right)$, where $\left(e_{i}\right)_{i \in \llbracket 1, d \rrbracket}$ denotes the canonical basis of $\mathbb{R}^{d}$, which corresponds to the cylindrical fractional Laplacian $\sum_{i=1}^{d}\left(\partial_{x_{i} x_{i}}^{2}\right)^{\frac{1}{2}}$ (cf. [3]).

In dimension one, (1.1) is investigated in the seminal paper [22]; the authors prove that (1.1) is well-posed if $b: \mathbb{R} \rightarrow \mathbb{R}$ is continuous and bounded and the exponent $\Phi: \mathbb{R}^{d} \rightarrow \mathbb{C}$ of the Lévy process $Z=\left(Z_{t}\right)$ verifies $(\operatorname{Re} \Phi(\lambda))^{-1}=O\left(|\lambda|^{-1}\right)$ as $|\lambda| \rightarrow \infty$. Note that, still in dimension one, uniqueness in law implies pathwise uniqueness. For $d \geq 1$ assuming that $\mu$ has a density, well-posedness of 11.1 follows by the results in [13].

Now, the operator which is at the moment only formally associated with the dynamics in (1.1) writes for all $\varphi \in C_{b}^{2}\left(\mathbb{R}^{d}\right)$, and $x \in \mathbb{R}^{d}$ :

$$
\begin{aligned}
\mathcal{L} \varphi(x) & =\text { p.v. } \int_{\mathbb{R}^{d} \backslash\{0\}}[\varphi(x+z)-\varphi(x)] \nu(d z)+\langle b(x), D \varphi(x)\rangle \\
& =L \varphi(x)+\langle b(x), D \varphi(x)\rangle,
\end{aligned}
$$

The current setting is said to be critical because, roughly speaking, the two terms in 1.5 have the same order one. Therefore it is not clear that the smoothing properties of the semi-group generated by the non-local operator are sufficient to regularize a transport term.

If the drift $b$ is itself Hölder continuous and bounded, stronger assumption than (C), then the well-posedness of the martingale problem for $\mathcal{L}$ can be established following 17. (see also Section 3 in [18). For unbounded Hölder drifts this property follows from the Schauder estimates established in 4 . All these results are based on perturbative techniques which exploit that the singularities of the corresponding heat-kernel serving as a proxy appearing in the analysis can be absorbed thanks to the Hölder continuity. However, under the sole continuity condition in $(\mathbf{C})$ those singularities can only be averaged. This is why in the current framework we will exploit $L^{p}$ estimates for singular kernels. We can for instance mention those of [10] in the more general degenerate Kolmogorov setting. We also remark that the proof of Lemma 4.34 (a Krylov's type estimate on the resolvent) seems to be of independent interest.

Results on weak well-posedness when $Z$ is non-degenerate symmetric stable with $\alpha>1$ and $b$ is in some $L^{p}$-spaces are available (see [11] and the references therein). We also mention 23] who investigates weak wellposedness when $\alpha<1$ for a subclass of symmetric stable processes $Z$ assuming that the $(1-\alpha)$-Hölder norm of $b$ is small enough.

Finally we note that equivalence between weak solutions to a class of SDEs driven by Poisson random measures and solutions to the martingale problem for a class of non-local operators is investigated in [16].

Let $\mathscr{D}\left(\mathbb{R}_{+}, \mathbb{R}^{d}\right)$ be the Skorokhod space of all càdlàg functions from $\mathbb{R}_{+}$into $\mathbb{R}^{d}$ endowed with the Skorokhod topology and consider the canonical process $\left(X_{t}\right), X_{t}(\omega)=\omega(t)$, for any $\omega \in \mathscr{D}\left(\mathbb{R}_{+}, \mathbb{R}^{d}\right)$. Let $\mathcal{L}$ be defined as in 1.5 .

Let us fix a Borel probability measure $\mu$ over $\mathbb{R}^{d}$. A solution to the $(\mathcal{L}, \mu)$-martingale problem is a probability measure $\mathbb{P}=\mathbb{P}^{\mu}$ on the Skorokhod space such that $\mathbb{P}\left(X_{0} \in B\right)=\mu(B), B \in \mathcal{B}\left(\mathbb{R}^{d}\right)$ and, moreover, for any function $\varphi \in C_{b}^{2}\left(\mathbb{R}^{d}\right)$,

$$
M_{t}=\varphi\left(X_{t}\right)-\varphi\left(X_{0}\right)-\int_{0}^{t} \mathcal{L} \varphi\left(X_{s}\right) d s, t \geq 0,
$$

is a $\mathbb{P}^{\mu}$-martingale (with respect to the canonical filtration). For a comprehensive study of such martingale problems see Chapter 4 in [7].

The martingale problem for $\mathcal{L}$ is well-posed, if for any initial distribution $\mu$ there exists a unique (in the sense of finite-dimensional distributions) solution to the $(\mathcal{L}, \mu)$-martingale problem. Our main result is the following one.

Theorem 1. Under (ND) and (C) the martingale problem for $\mathcal{L}(c f .(1.5)$ ) is well-posed.

\section{Main Steps for the Proof of Theorem 1}

Our approach is the following. Let us first assume that $b$ does not vary much, i.e.

$\left(\mathbf{C}_{\varepsilon}\right)$ Additionally to $(\mathbf{C})$, we assume that there exists $\varepsilon \in(0,1), b_{0} \in \mathbb{R}^{d}$ s.t.

$$
\left|b(x)-b_{0}\right| \leq \varepsilon, \quad x \in \mathbb{R}^{d} .
$$


The previous hypothesis means that we can choose $b_{0} \in \mathbb{R}^{d}$ and $\varepsilon>0$ small enough such that (2.6) holds. In this case, let us introduce, for any given starting point $x \in \mathbb{R}^{d}$, the following frozen proxy process:

$$
\tilde{X}_{t}^{x}=x+b_{0} t+Z_{t}, t \geq 0,
$$

where we recall that $\left(Z_{s}\right)_{s \geq 0}$ stands for a symmetric non-degenerate $d$-dimensional stable process of order $\alpha=1$ defined on some filtered probability space $\left(\Omega, \mathcal{F},\left(\mathcal{F}_{t}\right)_{t \geq 0}, \mathbb{P}\right)$. The generator of $\left(\tilde{X}_{t}^{x}\right)$ writes for all $\varphi \in C_{b}^{2}\left(\mathbb{R}^{d}\right)$ as:

$$
\begin{aligned}
\mathcal{L}^{b_{0}} \varphi(x)=\tilde{\mathcal{L}} \varphi(x) & =\text { p.v. } \int_{\mathbb{R}^{d} \backslash\{0\}}[\varphi(x+z)-\varphi(x)] \nu(d z)+\left\langle b_{0}, D \varphi(x)\right\rangle \\
& =L \varphi(x)+\left\langle b_{0}, D \varphi(x)\right\rangle, \quad x \in \mathbb{R}^{d},
\end{aligned}
$$

using again the notation 1.2 .

Using the regularity properties of the density of $Z_{t}$, under (ND), we find easily that $\tilde{X}_{t}^{x}$ admits for $t>0$ a $C^{\infty}$-density $\tilde{p}(t, x, \cdot)$ which can be expressed as:

$$
\tilde{p}(t, x, y)=p_{Z_{t}}\left(y-x-b_{0} t\right), \quad t>0 .
$$

Following the proof of Lemma 4.3 in [10] (see also Section 4.2 in [4]) we can as well show the following result (the sketch of the proof is postponed to Appendix).

Lemma 2.1 (Controls on the frozen density). The density $p_{Z_{t}}$ and its derivatives satisfy the following integrability properties. There exists a constant $C_{1}:=C_{1}((\mathbf{N D})) \geq 1$ s.t. for all multi-index $\beta,|\beta| \leq 2$,

$$
\left|\partial_{z}^{\beta} p_{Z_{t}}(z)\right| \leq \frac{C_{1}}{t^{|\beta|}} \bar{q}(t, z), \quad z \in \mathbb{R}^{d}
$$

where $\bar{q}(t, \cdot)$ is a probability density s.t. $t^{-d} \bar{q}\left(1, t^{-1} x\right)=\bar{q}(t, x)$, where $\bar{q}(1, \cdot) \in L^{p}\left(\mathbb{R}^{d}\right), p \in[1, \infty)$, and for all $\gamma<1$, there exists $C_{\gamma}$ s.t.

$$
\int_{\mathbb{R}^{d}}|z|^{\gamma} \bar{q}(t, z) \leq C_{\gamma} t^{\gamma}, \quad t>0
$$

Moreover, the density $\bar{q}$ enjoys the following property (cf. formula (4.26) in the proof of Lemma 4.3 in [10]): fix $K \geq 1$; there exists $C:=C(K)$ s.t. for all $t>0, x, y \in \mathbb{R}^{d}$ with $|y| / t \leq K$ then:

$$
\bar{q}(t, x+y) \leq C \bar{q}(t, x) .
$$

Also, for all $t>0, z \in \mathbb{R}^{d}, c_{0}>0, h \in \mathbb{R}^{d}$ with $|h| \leq c_{0} t$,

$$
\left|\partial_{z}^{\beta} p_{Z_{t}}(z+h)\right| \leq \frac{\tilde{C}}{t^{\beta}} \bar{q}(t, z), \tilde{C}:=\tilde{C}\left(C_{1}, c_{0}\right),\left|\Delta^{\frac{1}{2}} p_{Z_{t}}(z)\right| \leq \frac{C_{1}}{t} \bar{q}(t, z),
$$

and for all $\beta \in(0,1)$, there exists $C_{\beta} \geq 1$ s.t. for all $\left(z, z^{\prime}\right) \in\left(\mathbb{R}^{d}\right)^{2}$ :

$$
\left|\Delta^{\frac{1}{2}} p_{Z_{t}}(z)-\Delta^{\frac{1}{2}} p_{Z_{t}}\left(z^{\prime}\right)\right| \leq \frac{C_{\beta}}{t}\left(\frac{\left|z-z^{\prime}\right|}{t}\right)^{\beta}\left(\bar{q}(t, z)+\bar{q}\left(t, z^{\prime}\right)\right),
$$

as well as

$$
\left|D p_{Z_{t}}(z)-D p_{Z_{t}}\left(z^{\prime}\right)\right| \leq \frac{C_{\beta}}{t}\left(\frac{\left|z-z^{\prime}\right|}{t}\right)^{\beta}\left(\bar{q}(t, z)+\bar{q}\left(t, z^{\prime}\right)\right) .
$$

For a fixed parameter $\lambda>0$, we now introduce the resolvent associated with the frozen proxy process in 2.7). Namely, for all $x \in \mathbb{R}^{d}$ and $f \in C_{0}^{\infty}\left(\mathbb{R}^{d}\right)$ :

$$
\tilde{R}^{\lambda} \varphi(x):=\int_{0}^{+\infty} \exp (-\lambda t) \mathbb{E}\left[\left(\tilde{X}_{t}^{x}\right)\right] d t=\int_{0}^{+\infty} d t \exp (-\lambda t) \int_{\mathbb{R}^{d}} \tilde{p}(t, x, y) \varphi(y) d y .
$$

It is clear that the above function is the unique classical solution of the PDE:

$$
\tilde{\mathcal{L}} u(x)-\lambda u=-f(x), x \in \mathbb{R}^{d} .
$$

It also satisfies, for the smooth source considered, some Schauder estimates (see e.g. [18]). Eventually, we also derive from Lemma 2.1 the following important pointwise estimate. For all $p>d$ there exists $C_{p}$ s.t. for all $x \in \mathbb{R}^{d}$,

$$
\left|\tilde{R}^{\lambda} f(x)\right| \leq C_{p}\left(1+\lambda^{-1}\right)\|f\|_{L^{p}}, \quad f \in C_{0}^{\infty}\left(\mathbb{R}^{d}\right) .
$$


Indeed, from 2.10), one gets (using the Hölder inequality):

$$
\begin{aligned}
\left|\tilde{R}^{\lambda} f(x)\right| & \leq \int_{0}^{+\infty} d t \exp (-\lambda t) \int_{\mathbb{R}^{d}}|f(y)| \frac{C}{t^{d}} \bar{q}\left(1, \frac{y}{t}\right) d y \leq C \int_{0}^{+\infty} \frac{d t}{t^{\frac{d}{p}}} \exp (-\lambda t)\|f\|_{L^{p}} \\
& \leq C_{p}\left(1+\lambda^{-1}\right)\|f\|_{L^{p}} .
\end{aligned}
$$

We are actually interested in the corresponding PDE with variable coefficients involving $\mathcal{L}$ which writes:

$$
\mathcal{L} u(x)-\lambda u(x)=-f(x), x \in \mathbb{R}^{d} .
$$

The idea is to express a solution of $(2.19)$ in terms of the solutions of 2.17$)$ which are well understood. To this end we first write:

$$
\mathcal{L} \tilde{R}^{\lambda} f(x)-\lambda \tilde{R}^{\lambda} f(x)=-f(x)+\mathcal{R} f(x)=:-(I-\mathcal{R}) f(x), x \in \mathbb{R}^{d},
$$

where in the above equation the remainder operator $\mathcal{R}$ writes for all $f \in C_{0}^{\infty}\left(\mathbb{R}^{d}\right)$ :

$$
\mathcal{R} f(x)=(\mathcal{L}-\tilde{\mathcal{L}}) \tilde{R}^{\lambda} f(x)=\left\langle b(x)-b_{0}, D \tilde{R}^{\lambda} f(x)\right\rangle .
$$

The point is that a formal solution of $(2.19)$ is provided by the expression $\tilde{R}^{\lambda} \circ(I-\mathcal{R})^{-1} f(x)$ if $I-\mathcal{R}$ can be inverted on a suitable function space (see Section 4.1 for more details). A sufficient condition is that the remainder operator $\mathcal{R}$ has sufficiently small associated norm. Observe that for all $t>0$,

$$
\left|D \int_{\mathbb{R}^{d}} \tilde{p}(t, x, y) f(y) d y\right| \leq C t^{-1}\|f\|_{\infty} .
$$

Under our sole continuity conditions $\left(\mathbf{C}_{\varepsilon}\right)$, we cannot absorb such a time singularity pointwise (this could be done in the Hölder framework).

We are thus naturally in the framework of singular integrals, i.e. explosive contribution in time, for which one can expect the time singularity to be absorbed through averaging. The associated natural function spaces to be considered are thus the Lebesgue spaces $L^{p}\left(\mathbb{R}^{d}\right)$.

By Lemma 2.1 we will actually derive the following theorem which is the main technical tool to establish the well-posedness for 1.1 .

Theorem 2 ( $L^{p}$-estimates for the resolvent). Under (ND) we have, $\lambda>0$,

$$
\left\|D \tilde{R}^{\lambda} f\right\|_{L^{p}\left(\mathbb{R}^{d}\right)} \leq\left(1+\lambda^{-1}\right) C\left(p,(\mathbf{N D}), d,\left|b_{0}\right|\right)\|f\|_{L^{p}\left(\mathbb{R}^{d}\right)}, f \in C_{0}^{\infty}\left(\mathbb{R}^{d}\right) .
$$

We deduce

Theorem 3 ( $L^{p}$-estimates for the remainder in the critical case). Assume (ND) and $\left(\mathbf{C}_{\varepsilon}\right)$ hold. Let $\lambda \geq 1$. For any $p \in(1,+\infty)$, there exists $C_{p}:=C\left(p,(\mathbf{N D}), d,\|b\|_{\infty}\right)$ s.t.

$$
\|\mathcal{R} f\|_{L^{p}\left(\mathbb{R}^{d}\right)} \leq C_{p} \varepsilon\|f\|_{L^{p}\left(\mathbb{R}^{d}\right)}, \quad f \in C_{0}^{\infty}\left(\mathbb{R}^{d}\right) .
$$

Importantly, assuming $\left(\mathbf{C}_{\varepsilon}\right)$, we have: $\left|b_{0}\right| \leq\left|b_{0}-b(x)\right|+|b(x)| \leq 1+\|b\|_{\infty}$; hence the estimate in (2.23) is independent of $b_{0}$.

Theorem 3 gives that $(I-\mathcal{R})$ is invertible on $L^{p}\left(\mathbb{R}^{d}\right)$ if $\varepsilon$ is sufficiently small.

Proof. Write for all $x \in \mathbb{R}^{d}$ :

$$
|\mathcal{R} f(x)| \leq\left|b_{0}-b(x)\right|\left|D \tilde{R}^{\lambda} f(x)\right| \underset{2.6}{\leq} \varepsilon\left|D \tilde{R}^{\lambda} f(x)\right| .
$$

Hence,

$$
\|\mathcal{R} f\|_{L^{p}\left(\mathbb{R}^{d}\right)} \leq \varepsilon\left\|D \tilde{R}^{\lambda} f\right\|_{L^{p}\left(\mathbb{R}^{d}\right)} .
$$

Plugging 2.22 in 2.24 yields the result.

The previous estimates then allow to establish that the martingale problem associated with $\mathcal{L}$ is well-posed under the assumption $\left(\mathbf{C}_{\varepsilon}\right)$. From this first result, we can get rid of the almost constant coefficients through the continuity assumption and a localization argument. These points are detailed in Section 4 below. 


\section{Proof of the main $L^{p}$ estimates of Theorem 2}

To get the $L^{p}$-estimates in 2.22 , we will adopt the Coifmann and Weiss approach 6 as in [10]. It therefore suffices to establish the two following lemmas.

Lemma 3.1 (Global $L^{2}$-estimate). There exists a positive constant $C_{2}:=C_{2}((\mathbf{N D}))$ such that, for all $\lambda>0$ and for all $f \in L^{2}\left(\mathbb{R}^{d}\right)$,

$$
\left\|D \tilde{R}^{\lambda} f\right\|_{L^{2}\left(\mathbb{R}^{d}\right)} \leq C_{2}\|f\|_{L^{2}\left(\mathbb{R}^{d}\right)}
$$

We mention that this estimate would hold under weaker assumptions than (ND). In particular, no symmetry would a-priori be needed.

Lemma 3.2 (Deviation Controls). There exist constants $K$ and $C$ possibly depending on (ND) and $\left|b_{0}\right|$, s.t. for all $\xi, x \in \mathbb{R}^{d}$ the following control hold:

$$
\int_{0}^{+\infty} d t \exp (-\lambda t) \int_{|x-y| \geq K|x-\xi|}|D \tilde{p}(t, x, y)-D \tilde{p}(t, \xi, y)| d y \leq C\left(1+\lambda^{-1}\right) .
$$

Indeed, up to a direct symmetrization of the singular kernel involved, since the above estimates still hold for the adjoint operator, we readily derive that 2.22 follows from the controls of Lemmas 3.1 , 3.2 and Theorem 2.4 in Chapter III of [6]. To this purpose we also need an additional truncation procedure to separate the singular and non-singular part of the kernel (see e.g. Lemma 3.2 and eq. (3.11) in [10]).

Remark 3.1. We point out that, in the critical case the $L^{p}$-estimates for the fractional Laplacian or the gradient applied to the resolvent are the same. Indeed, in the Fourier space their symbol are respectively $|\xi|$ and $-i \xi$. The $L^{2}$-estimate will not make any difference and as far as the deviations are concerned, we recall from 2.15), (2.14) and (2.9) that both singular kernels share the same density estimate.

3.1. Proof of Lemma 3.1. Introduce first for $\eta>0$,

$$
\tilde{R}_{\eta}^{\lambda} f(x)=\int_{\eta}^{+\infty} d t \exp (-\lambda t) \int_{\mathbb{R}^{d}} \tilde{p}(t, x, y) f(y) d y
$$

We start from the representation of the density $\tilde{p}$ obtained under (ND) through Fourier inversion from 2.9 . Namely, for all $t>0,(x, y) \in\left(\mathbb{R}^{d}\right)^{2}$,

$$
\tilde{p}(t, x, y)=\frac{1}{(2 \pi)^{d}} \int_{\mathbb{R}^{d}} \exp \left(-i\left\langle\left(y-\left(x+b_{0} t\right)\right), p\right\rangle\right) \cdot \exp \left(-t \int_{\mathbb{S}^{d-1}}|\langle p, \xi\rangle| \mu(d \xi)\right) d p .
$$

Let $k=1, \ldots, d$ and $D_{k}=\partial_{x_{k}}$. We can compute, for $f \in \mathscr{S}\left(\mathbb{R}^{d}\right)$ (Schwartz class of $\mathbb{R}^{d}$ ), the Fourier transform:

$$
\begin{aligned}
\mathbb{R}^{d} \ni \zeta & \mapsto \mathcal{F}\left(D_{k} \tilde{R}_{\eta}^{\lambda} f\right)(\zeta)=\int_{\mathbb{R}^{d}} e^{i\langle\zeta, x\rangle} D_{k} \tilde{R}_{\eta}^{\lambda} f(x) d x=\left(-i \zeta_{k}\right) \mathcal{F}\left(\tilde{R}_{\eta}^{\lambda} f\right)(\zeta) \\
& =\left(-i \zeta_{k}\right) \int_{\mathbb{R}^{n}} e^{-i\langle\zeta, x\rangle}\left(\int_{\eta}^{+\infty} \exp (-\lambda t) \int_{\mathbb{R}^{d}} \tilde{p}(t, x, y) f(y) d y d t\right) d x
\end{aligned}
$$

Using the Fubini theorem and 2.9, we derive:

$$
\begin{aligned}
\mathcal{F}\left(D_{k} \tilde{R}_{\eta}^{\lambda} f\right)(\zeta)= & \left(-i \zeta_{k}\right) \int_{\eta}^{+\infty} \exp (-\lambda t) \int_{\mathbb{R}^{d}} \exp (-i\langle\zeta, y) f(y) \\
& \times\left(\int_{\mathbb{R}^{d}} \exp (-i\langle\zeta, x-y\rangle) p_{Z_{t}}\left(y-\left(x+b_{0} t\right)\right) d x\right) d y d t \\
= & \left(-i \zeta_{k}\right)\left(\int_{\eta}^{+\infty} \exp (-\lambda t) \int_{\mathbb{R}^{d}} \exp (-i\langle\zeta, y) f(y)\right. \\
& \exp \left(i t\left\langle\zeta, b_{0}\right\rangle\right) \times\left(\int_{\mathbb{R}^{d}} \exp (-i\langle\zeta, \tilde{x}\rangle) p_{Z_{t}}(-\tilde{x}) d \tilde{x}\right) d y d t
\end{aligned}
$$


setting $\tilde{x}=x+b_{0} t-y$ for the last identity. Recalling that by symmetry $p_{Z_{t}}(-\tilde{x})=p_{Z_{t}}(\tilde{x})$, we finally get

$$
\begin{aligned}
& \mathcal{F}\left(D_{k} \tilde{R}_{\eta}^{\lambda} f\right)(\zeta) \\
= & \left(-i \zeta_{k}\right) \int_{\eta}^{+\infty} \exp \left(t\left(-\lambda+i\left\langle\zeta, b_{0}\right\rangle\right)\right) \mathcal{F}(f)(\zeta) \mathcal{F}\left(p_{Z_{t}}\right)(t, \zeta) d t \\
= & \left(-i \zeta_{k}\right) \int_{\eta}^{+\infty} \exp \left(t\left(-\lambda+i\left\langle\zeta, b_{0}\right\rangle\right)\right) \mathcal{F}(f)(s, \zeta) \exp \left(-t \int_{\mathbb{S}^{d-1}}|\langle\zeta, \xi\rangle| \mu(d \xi)\right) d t,
\end{aligned}
$$

where $\left(\mathcal{F}(f)(\cdot), \mathcal{F}\left(p_{Z_{t}}\right)(\cdot)\right.$ denote the Fourier transforms of $f(\cdot), p_{Z_{t}}(\cdot)$.

Let us now compute $\left\|\mathcal{F}\left(D_{k} \tilde{R}_{\eta}^{\lambda} f\right)\right\|_{L^{2}\left(\mathbb{R}^{d}\right)}$. From the non degeneracy of $\mu$ we have:

$$
\left|\mathcal{F}\left(D_{k} \tilde{R}_{\eta}^{\lambda} f\right)(\zeta)\right| \leq C|\zeta| \int_{0}^{+\infty} \exp (-\lambda t)|\mathcal{F}(f)(\zeta)| \exp \left(-C^{-1} t|\zeta|\right) d t
$$

For the $L^{2}$-norm of $\mathcal{F}\left(D \tilde{R}_{\eta}^{\lambda} f\right)$, using the Cauchy-Schwarz inequality, we obtain:

$$
\begin{aligned}
\left\|\mathcal{F}\left(D_{k} \tilde{R}_{\eta}^{\lambda} f\right)\right\|_{L^{2}\left(\mathbb{R}^{d}\right)}^{2} \leq & C \int_{\mathbb{R}^{d}}\left(|\zeta| \int_{0}^{+\infty} \exp (-\lambda t)|\mathcal{F}(f)(\zeta)|^{2} \exp \left(-C^{-1} t|\zeta|\right) d t\right) \\
& \times\left(|\zeta| \int_{0}^{+\infty} \exp (-\lambda t) \exp \left(-C^{-1} t|\zeta|\right) d t\right) d \zeta \\
\leq & C \int_{\mathbb{R}^{d}}|\zeta| \int_{0}^{+\infty} \exp (-\lambda t)|\mathcal{F}(f)(\zeta)|^{2} \exp \left(-C^{-1} t|\zeta|\right) d t d \zeta \\
\leq & C \int_{\mathbb{R}^{d}}|\mathcal{F}(f)(\zeta)|^{2}\left(\int_{0}^{+\infty}|\zeta| \exp \left(-C^{-1} t|\zeta|\right) d t\right) d \zeta \\
\leq & C \int_{\mathbb{R}^{d}}|\mathcal{F}(f)(\zeta)|^{2} d \zeta=C\|\mathcal{F}(f)\|_{L^{2}\left(\mathbb{R}^{d}\right)} .
\end{aligned}
$$

The assertion now follows for $f \in \mathscr{S}\left(\mathbb{R}^{d}\right)$ from the Plancherel isometry, and for $f \in L^{2}\left(\mathbb{R}^{d}\right)$ by a density argument. From the uniformity of the previous controls, the final statement can eventually be derived letting $\eta$ go to zero through weak convergence arguments.

3.2. Proof of Lemma 3.2. Let us denote $\rho:=|x-y|, \gamma:=|x-\xi|$. From 2.13, 2.14) and the correspondence $2.9)$, we easily get that, for a fixed $\beta \in(0,1)$ :

$$
\begin{aligned}
& |D \tilde{p}(t, x, y)-D \tilde{p}(t, \xi, y)|=\left|D p_{Z_{t}}\left(y-x-b_{0} t\right)-D p_{Z_{t}}\left(y-\xi-b_{0} t\right)\right| \\
\leq & \frac{C_{\beta}}{t}\left(\frac{|x-\xi|}{t}\right)^{\beta}\left(\bar{q}\left(t, y-x-b_{0} t\right)+\bar{q}\left(t, y-\xi-b_{0} t\right)\right) \\
\leq & \frac{C_{\beta}}{t}\left(\frac{|x-\xi|}{t}\right)^{\beta}(\bar{q}(t, y-x)+\bar{q}(t, y-\xi))
\end{aligned}
$$

using as well the first bound in 2.13 for the last identity. Namely, a diagonal perturbation does not affect the density estimate. We therefore derive:

$$
\begin{aligned}
I & :=\int_{0}^{+\infty} d t \exp (-\lambda t) \int_{|x-y| \geq K|x-\xi|}|D \tilde{p}(t, x, y)-D \tilde{p}(t, \xi, y)| d y \\
& \leq C \int_{0}^{+\infty} d t \exp (-\lambda t) \int_{\rho>K \gamma} \frac{1}{t}\left(\frac{|x-\xi|}{t}\right)^{\beta}(\bar{q}(t, x-y)+\bar{q}(t, \xi-y)) d y . \\
& =: I_{1}+I_{2} .
\end{aligned}
$$

We can rewrite,

$$
I_{1}=C \gamma^{\beta} \int_{\rho>K \gamma} d y \int_{0}^{+\infty} d t\left(\mathbb{I}_{t \geq \gamma}+\mathbb{I}_{t<\gamma}\right) \frac{d t}{t^{1+\beta}} \exp (-\lambda t) \bar{q}(t, x-y)=: I_{11}+I_{12} .
$$


On the one hand, assuming w.l.o.g. that $\gamma \leq 1$, we get

$$
\begin{aligned}
I_{11} & \leq C \gamma^{\beta} \int_{\gamma}^{+\infty} d t \frac{\exp (-\lambda t)}{t^{1+\beta}} \int_{\mathbb{R}^{d}} \bar{q}(t, y-x) d y \leq C \gamma^{\beta}\left(\int_{\gamma}^{1} \frac{d t}{t^{1+\beta}}+\int_{1}^{\infty} \frac{d t}{\gamma^{\beta}} \exp (-\lambda t)\right) \\
& \leq C\left(1+\lambda^{-1}\right) .
\end{aligned}
$$

Write now, from the self-similarity properties of $\bar{q}$ and taking $0<\beta<\eta<1$ :

$$
\begin{aligned}
I_{12} & \leq C \gamma^{\beta} \int_{0}^{\gamma} d t \frac{\exp (-\lambda t)}{t^{1+\beta}} \int_{t|\tilde{y}| \geq K \gamma} \bar{q}(1, \tilde{y}) d \tilde{y} \\
& \leq C \gamma^{\beta}\left(\int_{0}^{\gamma} \frac{d t}{t^{1+\beta}} \int_{\frac{t|\tilde{y}|}{\gamma} \geq K}\left(K^{-1} \frac{t|\tilde{y}|}{\gamma}\right)^{\eta} q(1, \tilde{y}) d \tilde{y}\right) \\
& \leq C K^{-\eta} \gamma^{\beta-\eta} \int_{0}^{\gamma} \frac{d t}{t^{1+\beta-\eta}} \int_{\mathbb{R}^{d}}|\tilde{y}|^{\eta} \bar{q}(1, \tilde{y}) d \tilde{y} \leq \tilde{C} .
\end{aligned}
$$

Let us now turn to $I_{2}$ in 3.28 .

$$
I_{2}=C \gamma^{\beta} \int_{0}^{+\infty} \frac{d t}{t^{1+\beta}} \exp (-\lambda t)\left(\mathbb{I}_{t>\gamma}+\mathbb{I}_{t \leq \gamma}\right)\left(\int_{\mathbb{R}^{d}} \mathbb{I}_{\rho>K \gamma} \bar{q}(t, \xi-y) d y\right)=: I_{21}+I_{22} .
$$

The contribution $I_{21}$, can be handled as $I_{11}$ introduced in $(3.29)$ and therefore also satisfies 3.30 . To analyze $I_{22}$, we first set $z=t^{-1}(\xi-y)$ and recall that:

$$
K \gamma<\rho=|x-y| \leq|\xi-y|+|x-\xi| \leq|\xi-y|+\gamma \Rightarrow|\xi-y| \geq(K-1) \gamma,
$$

from which we deduce that $I_{22}$ can be handled as $I_{12}$ above up to a modifications of the considered constants. Hence, it also satisfies 3.31). Namely, we eventually have from 3.30 and 3.31 that for all $i \in\{1,2\}$, $I_{i} \leq C\left(1+\lambda^{-1}\right)$, which plugged into 3.28 gives the statement.

\section{WELL-POSEDNESS OF THE MARTINGALE PROBLEM}

We prove in this section our Theorem 1 . First under the local condition $\left(\mathbf{C}_{\varepsilon}\right)$ and then extend it through the continuity assumption through a localization argument.

Existence of a solution to the martingale problem for $(\mathcal{L}, \mu)$, for any initial distribution $\mu$, can be proved, under (ND) and (C), applying, for instance, Theorem 4.1 in [15. Such theorem is based on Theorem 4.5 .4 in [7] about relations between the positive maximum principle and the existence of solutions to the martingale problem. Theorem 4.5.4 has been used to prove existence results for martingale problems associated to pseudodifferential operators with symbols $\Phi(x, \lambda)$ which are continuous in $x$. We only mention here [8] and [15] (see also the references therein).

Thus in the sequel we concentrate on the problem of uniqueness.

4.1. Uniqueness of the martingale problem under $\left(\mathbf{C}_{\varepsilon}\right)$. Fix a Borel probability distribution $\mu$ on $\mathbb{R}^{d}$. Let $\mathbb{P}^{\mu}$ be any solution to the $(\mathcal{L}, \mu)$-martingale problem and denote by $\left(X_{t}\right)_{t \geq 0}$ the associated canonical process on the Skorokhod space. Moreover define $\mathbb{E}^{\mathbb{P}^{\mu}}=\mathbb{E}^{\mu}$.

To derive uniqueness we will crucially rely on the following Krylov type estimate whose proof is postponed to the end of the section.

Lemma 4 (Krylov type bound). Let $\mathbb{P}^{\mu}$ be any solution to the $(\mathcal{L}, \mu)$-martingale problem. Define the corresponding resolvent

$$
G(\lambda) f=\mathbb{E}^{\mu}\left[\int_{0}^{+\infty} \exp (-\lambda s) f\left(X_{s}\right) d s\right]
$$

Then for all $f \in C_{0}^{\infty}\left(\mathbb{R}^{d}\right), \lambda \geq 1$, and for all $p>d$ there exists $C:=C\left(p, d, \varepsilon,\|b\|_{\infty}\right)>0$ s.t.

$$
|G(\lambda) f| \leq C\|f\|_{L^{p}}
$$

Observe that the above bound actually provides by duality that $G(\lambda)$ possesses a density $p^{\lambda}=p^{\lambda, \mu}$ which belongs to $L^{q}\left(\mathbb{R}^{d}\right), p^{-1}+q^{-1}=1$. Lemma 4 precisely provides a good tool to derive uniqueness. 
4.1.1. Derivation of uniqueness. We will apply Theorem 4.4.2 in [7] (see also Theorem 6.2.3 in [21]). This says that if two solutions $\mathbb{P}_{1}$ and $\mathbb{P}_{2}$ of the $(\mathcal{L}, \mu)$-martingale problem have the same one-dimensional marginal distributions then they coincide (i.e., they have the same finite-dimensional distributions).

Let $\mathbb{P}^{\mu}$ be any solution to the $(\mathcal{L}, \mu)$-martingale problem. Let $\lambda \geq 1$. For $f \in C_{0}^{\infty}\left(\mathbb{R}^{d}\right)$ consider the resolvent $\tilde{R}^{\lambda} f(x)$ introduced in $[2.16)$. This is a smooth function (see e.g. [18]). Set $\varphi(x)=\tilde{R}^{\lambda} f(x)$. The idea is now to expand, $\varphi\left(X_{t}\right):=\tilde{R}^{\lambda} f\left(X_{t}\right)$. We know that

$$
\varphi\left(X_{t}\right)-\varphi\left(X_{0}\right)-\int_{0}^{t} \mathcal{L} \varphi\left(X_{s}\right) d s=\tilde{R}^{\lambda} f\left(X_{t}\right)-\tilde{R}^{\lambda} f\left(X_{0}\right)-\int_{0}^{t} \mathcal{L} \tilde{R}^{\lambda} f\left(X_{s}\right) d s
$$

is a $\mathbb{P}^{\mu}$-martingale. We write:

$$
\begin{aligned}
& \mathbb{E}^{\mu}\left[\tilde{R}^{\lambda} f\left(X_{t}\right)\right]-\mathbb{E}^{\mu} \tilde{R}^{\lambda} f\left(X_{0}\right)=\mathbb{E}^{\mu}\left[\int_{0}^{t}(\mathcal{L}-\lambda) \tilde{R}^{\lambda} f\left(X_{s}\right) d s\right]+\lambda \mathbb{E}^{\mu}\left[\int_{0}^{t} \tilde{R}^{\lambda} f\left(X_{s}\right) d s\right] \\
= & -\mathbb{E}^{\mu}\left[\int_{0}^{t} f\left(X_{s}\right) d s\right]+\mathbb{E}^{\mu}\left[\int_{0}^{t}(\mathcal{L}-\tilde{\mathcal{L}}) \tilde{R}^{\lambda} f\left(X_{s}\right) d s\right]+\lambda \mathbb{E}^{\mu}\left[\int_{0}^{t} \tilde{R}^{\lambda} f\left(X_{s}\right) d s\right] .
\end{aligned}
$$

Integrating over $[0, \infty)$ with respect to $e^{-\lambda t} d t$ and using the Fubini theorem we find

$$
\begin{aligned}
\mathbb{E}^{\mu} \tilde{R}^{\lambda} f\left(X_{0}\right) & =\mathbb{E}^{\mu}\left[\int_{0}^{+\infty} \exp (-\lambda s) f\left(X_{s}\right) d s\right]-\mathbb{E}^{\mu}\left[\int_{0}^{+\infty} \exp (-\lambda s)(\mathcal{L}-\tilde{\mathcal{L}}) \tilde{R}^{\lambda} f\left(X_{s}\right) d s\right] \\
& =\mathbb{E}^{\mu}\left[\int_{0}^{+\infty} \exp (-\lambda s)(I-\mathcal{R}) f\left(X_{s}\right) d s\right]
\end{aligned}
$$

where we have used the remainder operator $\mathcal{R}$ which writes for all $f \in C_{0}^{\infty}\left(\mathbb{R}^{d}\right)$ :

$$
\mathcal{R} f(x)=(\mathcal{L}-\tilde{\mathcal{L}}) \tilde{R}^{\lambda} f(x)=\left\langle b(x)-b_{0}, D \tilde{R}^{\lambda} f(x)\right\rangle .
$$

Let now $\mathbb{P}_{1}$ and $\mathbb{P}_{2}$ be two solutions for the $(\mathcal{L}, \mu)$-martingale problem. Let $G_{i}(\lambda) f=\mathbb{E}^{\mathbb{P}_{i}}\left[\int_{0}^{+\infty} \exp (-\lambda s) f\left(X_{s}\right) d s\right]$, $i=1,2$. We find by 4.35

$$
G_{i}(\lambda) f=\mathbb{E}^{\mu} \tilde{R}^{\lambda} f\left(X_{0}\right)+G_{i}(\lambda) \mathcal{R} f .
$$

Define $T(\lambda): C_{0}^{\infty}\left(\mathbb{R}^{d}\right) \rightarrow \mathbb{R}, T(\lambda) f=G_{1}(\lambda) f-G_{2}(\lambda) f$. We have

$$
T(\lambda)(I-\mathcal{R}) f=0, \quad f \in C_{0}^{\infty}\left(\mathbb{R}^{d}\right) .
$$

By using Lemma 4 we know that $T(\lambda)$ can be extended to a bounded linear operator from $L^{p}\left(\mathbb{R}^{d}\right.$ ) into $\mathbb{R}$ (we still denote by $T(\lambda)$ such extension). By Theorem 3 we know that $I-\mathcal{R}$ is invertible on $L^{p}\left(\mathbb{R}^{d}\right)$ for $p>d$ (under assumption $\left(\mathbf{C}_{\varepsilon}\right)$ ). Hence choosing $\varepsilon$ small enough (independently of $\lambda \geq 1$ ) we find $\|T(\lambda)\|_{L\left(L^{p}\left(\mathbb{R}^{d}\right) ; \mathbb{R}\right)}=0$, $\lambda \geq 1$.

We obtain that $\mathbb{E}^{\mathbb{P}_{1}} f\left(X_{t}\right)=\mathbb{E}^{\mathbb{P}_{2}} f\left(X_{t}\right), t \geq 0$, for any $f \in C_{0}^{\infty}\left(\mathbb{R}^{d}\right)$, by using the uniqueness of the Laplace transform. By a standard approximation procedure we also get, for any Borel set $B \subset \mathbb{R}^{d}$,

$$
\mathbb{P}_{1}\left(X_{t} \in B\right)=\mathbb{P}_{2}\left(X_{t} \in B\right), \quad t \geq 0 .
$$

4.1.2. Proof of Lemma 4. We will adapt an approximation technique of resolvents introduced by N.V. Krylov in the gaussian setting (cf. [14] and Chapter VI in [1]).

Arguing as in Theorem 2.1 of [12] (see also Theorem 3.1 in [5] and Section 3 in [16]) one proves that on some stochastic basis $\left(\Omega, \mathcal{F},\left(\mathcal{F}_{t}\right), \mathbb{P}\right)$ there exists a $d$-dimensional 1 -stable process $Z$ as in $(1.1)$, an $\mathcal{F}_{0}$-measurable r.v. $X_{0}$ with law $\mu$ and a solution $Y=\left(Y_{t}\right)$ to

$$
Y_{t}=X_{0}+\int_{0}^{t} b\left(Y_{s}\right) d s+Z_{t}, \quad t \geq 0
$$

such that the law of $Y$ coincides with $\mathbb{P}^{\mu}$ on the Skorokhod space.

Fix $\lambda \geq 1$. For any Borel set $C \subset \mathbb{R}^{d}$ the measure

$$
\gamma(C)=\mathbb{E} \int_{0}^{\infty} e^{-\lambda t} 1_{C}\left(Y_{t}\right) d t
$$

is well-defined. Moreover for any ball $B(z, r)$ we have

$$
\gamma(B(z, r))>0, z \in \mathbb{R}^{d}, r>0 .
$$


We argue by contradiction. If for some ball $B=B(z, r)$ we have $\gamma(B)=0$, then for any $T>0$ there exists $0<t<T$ such that

$$
\mathbb{E}\left[1_{B}\left(Y_{t}\right)\right]=\mathbb{P}\left(Y_{t} \in B\right)=0 .
$$

We choose $T>0$ such that $T\|b\|_{\infty}<r / 2$. There exists $0<t<T$ such that $\mathbb{P}\left(\left|Y_{t}-z\right|<r\right)=0$. We find

$$
\begin{aligned}
\mathbb{P}\left(\left|Y_{t}-z\right|<r\right) & \geq \mathbb{P}\left(\left|\int_{0}^{t} b\left(Y_{s}\right) d s\right|<r / 2,\left|Z_{t}+X_{0}-z\right|<r / 2\right) \\
& =\mathbb{P}\left(\left|Z_{t}+X_{0}-z\right|<r / 2\right)>0,
\end{aligned}
$$

because $X_{0}$ is independent of $Z_{t}$ and the support of the distribution of $Z_{t}$ is $\mathbb{R}^{d}$ (see, for instance, Theorem 3.4 with $A=0$ in [19]). We have found a contradiction and so (4.38) holds.

Recall that $\mathbb{E}^{\mu}$ denotes expectation with respect to $\mathbb{P}^{\mu}$ and $\left(X_{t}\right)$ is the canonical process. As before we consider the measure $\gamma=\gamma^{\mu, \lambda}$ :

$$
\gamma(C)=\mathbb{E}^{\mu} \int_{0}^{\infty} e^{-\lambda t} 1_{C}\left(X_{t}\right) d t, \quad C \in \mathcal{B}\left(\mathbb{R}^{d}\right) .
$$

Now we introduce $\phi(x)=c_{d}\left(1+|x|^{d+1}\right)^{-1}, x \in \mathbb{R}^{d}$, with $c_{d}=\left(\int_{\mathbb{R}^{d}} \phi(x) d x\right)^{-1}$. Note the following bound on the first and second derivatives of $\phi$ :

$$
|D \phi(x)|+\left\|D^{2} \phi(x)\right\|_{\mathbb{R}^{d} \otimes \mathbb{R}^{d}} \leq C_{d} \phi(x), \quad x \in \mathbb{R}^{d} .
$$

For $\delta>0$, we consider $\phi_{\delta}(x)=\frac{1}{\delta^{d}} \phi(x / \delta), x \in \mathbb{R}^{d}$. Using $\phi_{\delta}(x-y) \geq \frac{1}{2} 1_{B(x, \delta)}(y)$ and 4.38 we can define

$$
b_{\delta}(x)=\frac{\int_{\mathbb{R}^{d}} \phi_{\delta}(x-y) b(y) \gamma(d y)}{\int_{\mathbb{R}^{d}} \phi_{\delta}(x-y) \gamma(d y)}, x \in \mathbb{R}^{d}, \quad \delta>0 .
$$

Using 4.39 it is straightforward to check that $b_{\delta} \in C_{b}^{2}\left(\mathbb{R}^{d}, \mathbb{R}^{d}\right)$, i.e. $b_{\delta}$ has first and second bounded and continuous derivatives, $\delta>0$. Moreover $\left\|D b_{\delta}\right\|_{\infty} \leq \frac{c}{\delta}$, $\left\|D^{2} b_{\delta}\right\|_{\infty} \leq \frac{c}{\delta^{2}}$.

Let now $\varphi \in C_{b}^{2}\left(\mathbb{R}^{d}\right)$; by the martingale property:

$$
\left.\mathbb{E}^{\mu} \varphi\left(X_{t}\right)=\mathbb{E}^{\mu} \varphi\left(X_{0}\right)+\mathbb{E}^{\mu} \int_{0}^{t}\left[\mathcal{L} \varphi\left(X_{s}\right)-\lambda \varphi\left(X_{s}\right)\right] d s+\mathbb{E}^{\mu} \lambda \int_{0}^{t} \varphi\left(X_{s}\right)\right] d s .
$$

Integrating over $[0, \infty)$ with respect to $e^{-\lambda t} d t$ and using the Fubini theorem we find

$$
\mathbb{E}^{\mu} \varphi\left(X_{0}\right)=\int_{\mathbb{R}^{d}}[\lambda \varphi(y)-\mathcal{L} \varphi(y)] \gamma(d y) .
$$

Now we replace $\varphi$ by the convolution $\varphi * \phi_{\delta}$ so that (using also 4.44)

$$
\begin{gathered}
\mathbb{E}^{\mu}\left[\varphi * \phi_{\delta}\left(X_{0}\right)\right]=\int_{\mathbb{R}^{d}}\left[\lambda \varphi * \phi_{\delta}(y)-\mathcal{L}\left[\varphi * \phi_{\delta}\right](y)\right] \gamma(d y) \\
=\int_{\mathbb{R}^{d}}\left[\lambda \varphi * \phi_{\delta}(y)-L \varphi * \phi_{\delta}(y)\right] \gamma(d y)-\int_{\mathbb{R}^{d}} D \varphi(z) \cdot \int_{\mathbb{R}^{d}} \phi_{\delta}(z-y) b(y) \gamma(d y) d z \\
=\int_{\mathbb{R}^{d}}\left[\lambda \varphi * \phi_{\delta}(y)-L \varphi * \phi_{\delta}(y)\right] \gamma(d y)-\int_{\mathbb{R}^{d}} D \varphi(z) \cdot b_{\delta}(z) \int_{\mathbb{R}^{d}} \phi_{\delta}(z-y) \gamma(d y) d z \\
=\int_{\mathbb{R}^{d}} \int_{\mathbb{R}^{d}}\left(\lambda \varphi(p)-\left[b_{\delta} \cdot D+L\right] \varphi(p)\right) \phi_{\delta}(p-y) \gamma(d y) d p .
\end{gathered}
$$

Now we consider the operator

$$
\mathcal{L}_{\delta}=b_{\delta} \cdot D+L
$$

and take $\varphi=G_{\delta}(\lambda) f, f \in C_{0}^{\infty}\left(\mathbb{R}^{d}\right)$, where $G_{\delta}(\lambda)$ is the resolvent of the martingale solution $\mathbb{P}^{\delta, x}$ starting at $\delta_{x}$ associated to the operator $\mathcal{L}_{\delta}$ :

$$
G_{\delta}(\lambda) f(x)=\mathbb{E}^{\delta, x}\left[\int_{0}^{+\infty} \exp (-\lambda s) f\left(X_{s}\right) d s\right], \quad x \in \mathbb{R}^{d} .
$$

Since $b_{\delta} \in C_{b}^{2}\left(\mathbb{R}^{d}, \mathbb{R}^{d}\right)$ it is not difficult to check that that $\lambda G_{\delta}(\lambda) f(p)-\left[b_{\delta} \cdot D+L\right] G_{\delta}(\lambda) f(p)=f(p), p \in \mathbb{R}^{d}$. It follows that

$$
\mathbb{E}^{\mu}\left[G_{\delta}(\lambda) f * \phi_{\delta}\left(X_{0}\right)\right]=\int_{\mathbb{R}^{d}} f * \phi_{\delta}(y) \gamma(d y) .
$$


Hence we get the crucial approximation result:

$$
\mathbb{E}^{\mu}\left[G_{\delta}(\lambda) f * \phi_{\delta}\left(X_{0}\right)\right] \rightarrow \mathbb{E}^{\mu} \int_{0}^{\infty} e^{-\lambda t} f\left(X_{t}\right) d t, \quad \delta \rightarrow 0^{+},
$$

since $\left(f * \phi_{\delta}\right)$ is uniformly bounded and converges pointwise to $f$ on $\mathbb{R}^{d}$.

Now we consider 4.35 which we write for the solution $\mathbb{P}^{\delta, x}$ to the martingale problem for $\mathcal{L}_{\delta}$ (starting at the delta Dirac in $x)$. We write $\mathcal{R}^{\delta} f(x)=\left\langle b_{\delta}(x)-b_{0}, D \tilde{R}^{\lambda} f(x)\right\rangle$ and note that

$$
\left|b_{\delta}(x)-b_{0}\right|<\varepsilon, \quad x \in \mathbb{R}^{d}, \delta>0 .
$$

Similarly to 4.35 we find

$$
\tilde{R}^{\lambda} f(x)=\mathbb{E}^{\delta, x} \int_{0}^{+\infty} \exp (-\lambda s) \int_{\mathbb{R}^{d}}\left(I-\mathcal{R}^{\delta}\right) f\left(X_{s}\right) d s .
$$

Since $b_{\delta} \in C_{b}^{2}\left(\mathbb{R}^{d}, \mathbb{R}^{d}\right)$ it is known that the resolvent $G_{\delta}(\lambda) f(x)=\mathbb{E}^{\delta, x}\left[\int_{0}^{+\infty} d s \exp (-\lambda s) f\left(X_{s}\right)\right]$ has a density $p^{\lambda, \delta}(x, y)$, i.e.,

$$
G_{\delta}(\lambda) f(x)=\int_{\mathbb{R}^{d}} f(y) p^{\lambda, \delta}(x, y) d y, \quad x \in \mathbb{R}^{d} .
$$

Moreover, $p^{\lambda, \delta}(x, \cdot) \in L^{q}\left(\mathbb{R}^{d}\right), q>1$. This follows from the estimate

$$
\left|G_{\delta}(\lambda) f(x)\right| \leq C_{\delta}\|f\|_{L^{p}\left(\mathbb{R}^{d}\right)}, \quad f \in C_{0}^{\infty}\left(\mathbb{R}^{d}\right), \delta>0
$$

see Lemma A.1. It follows that

$$
\tilde{R}^{\lambda} f(x)=\int_{\mathbb{R}^{d}}\left(I-\mathcal{R}^{\delta}\right) f(y) p^{\lambda, \delta}(x, y) d y .
$$

Recall from 2.18 that for all $p>d$ there exists $C_{p}$ s.t. for all $\mathbf{x} \in \mathbb{R}^{d}$

$$
\left|\tilde{R}^{\lambda} f(x)\right| \leq C_{p}\left(1+\lambda^{-1}\right)\|f\|_{L^{p}\left(\mathbb{R}^{d}\right)}, \quad f \in C_{0}^{\infty}\left(\mathbb{R}^{d}\right) .
$$

Moreover, by Theorem 3 we know that $I-\mathcal{R}^{\delta}$ is invertible on $L^{p}\left(\mathbb{R}^{d}\right)$ for $p>d$. For any $g \in L^{p}\left(\mathbb{R}^{d}\right)$ we find

$$
\begin{gathered}
G_{\delta}(\lambda) g(x)=\int_{\mathbb{R}^{d}} g(y) p^{\lambda, \delta}(x, y) d y \\
\leq C_{p}\left(1+\lambda^{-1}\right)\left\|\left(I-\mathcal{R}^{\delta}\right)^{-1} g\right\|_{L^{p}} \leq \tilde{C}_{p}\left(1+\lambda^{-1}\right)\|g\|_{L^{p}} .
\end{gathered}
$$

It follows that, for $\delta>0$,

$$
\left|\mathbb{E}^{\mu}\left[G_{\delta}(\lambda) f * \phi_{\delta}\left(X_{0}\right)\right]\right| \leq \tilde{C}_{p}\left(1+\lambda^{-1}\right)\|f\|_{L^{p}}
$$

$f \in C_{0}^{\infty}\left(\mathbb{R}^{d}\right)$. Passing to the limit as $\delta \rightarrow 0^{+}$we get the assertion by 4.41).

4.2. Uniqueness of the martingale problem under (C) by a localization argument. We will use Theorem 4.6.2 in [7.

First recall that an operator like $\mathcal{L}$ under assumption $\left(\mathbf{C}_{\varepsilon}\right)$ has the property that the associated martingale problem is well-posed for any initial distribution $\mu$.

By Theorem 4.6.1 in [7, such operator under $\left(\mathbf{C}_{\varepsilon}\right)$ has the following additional property: for any initial distribution $\mu$ and for any open set $U \subset \mathbb{R}^{d}$ there exists a unique in law probability measure $\mathbb{P}$ on $\mathscr{D}\left(\mathbb{R}_{+}, \mathbb{R}^{d}\right)$ such that $\mathbb{P}\left(X_{0} \in B\right)=\mu(B), B \in \mathcal{B}\left(\mathbb{R}^{d}\right)$,

$$
X(\cdot)=X(\cdot \wedge \tau), \quad \mathbb{P} \text {-a.s., where } \tau=\tau^{U}=\inf \left\{t \geq 0: X_{t} \notin U\right\}
$$

( $\tau=+\infty$ if the set is empty) is a stopping time with respect to the canonical filtration, and finally $\varphi\left(X_{t \wedge \tau}\right)-$ $\int_{0}^{t \wedge \tau} \mathcal{L} \varphi\left(X_{s}\right) d s, t \geq 0, \varphi \in C_{b}^{2}\left(\mathbb{R}^{d}\right)$, is a martingale with respect to the canonical filtration (recall that $\left(X_{t}\right)=$ $(X(t))$ indicates the canonical process).

One says that under assumption $\left(\mathbf{C}_{\varepsilon}\right)$ for any open set $U \subset \mathbb{R}^{d}$, for any initial distribution $\mu$ the stopped martingale problem for $(\mathcal{L}, U, \mu)$ is well-posed.

Now only assuming $(\mathbf{C})$, we construct a suitable covering $\left(U_{j}\right)_{j \geq 1}$ of open sets in $\mathbb{R}^{d}$ such that for any initial distribution $\mu$ the stopped martingale problem for $\left(\mathcal{L}, U_{j}, \mu\right)$ is well-posed, for any $j \geq 1$. According to Theorem 4.6.2 in [7] we conclude that the (global) martingale problem for $\mathcal{L}$ is well-posed. 
To construct such covering of $\mathbb{R}^{d}$ we note that by the continuity of $b$ we can find a sequence $\left(x_{j}\right) \subset \mathbb{R}^{d}$, $j \geq 1$, and numbers $\delta_{j}>0$ such that the open balls $U_{j}=B\left(x_{j}, \delta_{j}\right)$ of center $x_{j}$ and radius $\delta_{j}$ form a covering for $\mathbb{R}^{d}$ and moreover we have $\left|b(x)-b\left(x_{j}\right)\right|<\varepsilon$ (cf. (2.6)) for any $x \in B\left(x_{j}, 2 \delta_{j}\right), j \geq 1$.

The balls $\left\{B\left(x_{j}, \delta_{j}\right)\right\}_{j \geq 1}$ give the required covering $\left\{U_{j}\right\}_{j \geq 1}$. Let us define operators $\mathcal{L}_{j}$ such that

$$
\mathcal{L}_{j} \varphi(x)=\mathcal{L} \varphi(x), \quad x \in U_{j}, \quad \varphi \in C_{b}^{2}\left(\mathbb{R}^{d}\right),
$$

and such that each $\mathcal{L}_{j}$ verifies $\left(\mathbf{C}_{\varepsilon}\right)$. We fix $j \geq 1$ and consider $\eta_{j} \in C_{0}^{\infty}\left(\mathbb{R}^{d}\right)$ with $0 \leq \eta_{j} \leq 1, \eta_{j}=1$ in $B\left(x_{j}, \delta_{j}\right)$ and $\eta_{j}=0$ outside $B\left(x_{j}, 2 \delta_{j}\right)$. Now define

$$
b^{j}(x):=\eta_{j}(x) b(x)+\left(1-\eta_{j}(x)\right) b\left(x_{j}\right) .
$$

It is easy to see that $b^{j}(x)=b(x), x \in U_{j}$ and $\left|b^{j}(x)-b\left(x_{j}\right)\right|<\varepsilon$, for any $x \in \mathbb{R}^{d}$. Let us consider

$$
\mathcal{L}_{j} \varphi(x)=\text { p.v. } \int_{\mathbb{R}^{d} \backslash\{0\}}[\varphi(x+z)-\varphi(x)] \nu(d z)+\left\langle b^{j}(x), D \varphi(x)\right\rangle .
$$

Such operators verifies $\left(\mathbf{C}_{\varepsilon}\right)$ and so by the first part of the proof, for any initial distribution $\mu$ the stopped martingale problem for $\left(\mathcal{L}_{j}, U_{j}, \mu\right)$ is well-posed, for any $j \geq 1$. Thanks to 4.45 the stopped martingale problem for $\left(\mathcal{L}, U_{j}, \mu\right)$ is also well-posed, for any $j \geq 1$. This finishes the proof.

\section{Appendix A. Proof of Lemma 2.1}

We recall that we here aim at controlling the density and its derivatives of the random variable $Z_{t}$ where $Z$ is a stable process of index $\alpha=1$ satisfying the non-degeneracy condition (1.4) in assumption (ND).

Let us recall that, for a given fixed $t>0$, we can use an Itô-Lévy decomposition at the associated characteristic stable time scale (i.e. the truncation is performed at the threshold $t$ ) to write $Z_{t}:=M_{t}^{t}+N_{t}^{t}$ where $M_{t}^{t}$ and $N_{t}^{t}$ are independent random variables. More precisely,

$$
N_{s}^{t}=\int_{0}^{s} \int_{|x|>t} x P(d u, d x), \quad M_{s}^{t}=Z_{s}-N_{s}^{t}, \quad s \geq 0,
$$

where $P$ is the Poisson random measure associated with the process $Z$; for the considered fixed $t>0, M_{t}^{t}$ and $N_{t}^{t}$ correspond to the small jumps part and large jumps part respectively w.r.t. the corresponding typical scale of order $t$. A similar decomposition has been already used in the literature (see, for instance the proof of Lemma 4.3 in [10] and the references therein). It is useful to note that the cutting threshold in A.46 precisely yields for the considered $t>0$ that:

$$
N_{t}^{t} \stackrel{\text { (law) }}{=} t N_{1}^{1} \text { and } M_{t}^{t} \stackrel{(\text { law })}{=} t M_{1}^{1} .
$$

To check the assertion about $N^{t}$ we start with

$$
\mathbb{E}\left[e^{i\left\langle p, N_{t}^{t}\right\rangle}\right]=\exp \left(t \int_{\mathbb{S}^{d-1}} \int_{t}^{\infty}(\cos (\langle p, r \theta\rangle)-1) \frac{d r}{r^{2}} \tilde{\mu}(d \theta)\right), \quad p \in \mathbb{R}^{d}
$$

(see $\left[1.2\right.$ and [20]). Changing variable to $\frac{r}{t}=s$ we get that $\mathbb{E}\left[e^{i\left\langle p, N_{t}^{t}\right\rangle}\right]=\mathbb{E}\left[e^{i\left\langle p, t N_{1}^{1}\right\rangle}\right]$ for any $p \in \mathbb{R}^{d}$ and this shows the assertion (similarly we get the statement for $M$ ). The density of $Z_{t}$ then writes

$$
p_{Z_{t}}(x)=\int_{\mathbb{R}^{d}} p_{M_{t}^{t}}(x-\xi) P_{N_{t}^{t}}(d \xi),
$$

where $p_{M_{t}^{t}}(\cdot)$ corresponds to the density of $M_{t}^{t}$ and $P_{N_{t}^{t}}$ stands for the law of $N_{t}^{t}$. From Lemma A.2 in [10] (see as well Lemma B.1 in $[9]), p_{M_{t}^{t}}(\cdot)$ belongs to the Schwartz class $\mathscr{S}\left(\mathbb{R}^{d}\right)$ and satisfies that for all $m \geq 1$ and all multi-index $\beta,|\beta| \leq 2$, there exist constants $\bar{C}_{m}, C_{m}$ s.t. for all $t>0, x \in \mathbb{R}^{d}$ :

$$
\left|D_{x}^{\beta} p_{M_{t}^{t}}(x)\right| \leq \frac{\bar{C}_{m}}{t^{\ell}} p_{\bar{M}}(t, x) \text {, where } p_{\bar{M}}(t, x):=\frac{C_{m}}{t^{d}}\left(1+\frac{|x|}{t}\right)^{-m},
$$

where $C_{m}$ is chosen in order that $p_{\bar{M}}(t, \cdot)$ be a probability density.

We carefully point out that, to establish the indicated results, since we are led to consider potentially singular spherical measures, we only focus on integrability properties similarly to [10. The main idea thus consists in exploiting A.46), A.48 and A.49). The derivatives on which we want to obtain quantitative bounds will be expressed through derivatives of $p_{M_{t}^{t}}(\cdot)$, which also give the corresponding time singularities. However, as for general stable processes, the integrability restrictions come from the large jumps (here $N_{t}^{t}$ ) and only 
depend on its stability index here equal to 1. A crucial point then consists in observing that the convolution $\int_{\mathbb{R}^{d}} p_{\bar{M}}(t, x-\xi) P_{N_{t}^{t}}(d \xi)$ actually corresponds to the density of the random variable

$$
\bar{Z}_{t}:=\bar{M}_{t}+N_{t}^{t}, t>0
$$

(where $\bar{M}_{t}$ has density $p_{\bar{M}}(t,$.$) and is independent of N_{t}^{t}$; to have such decomposition one can define each $\bar{Z}_{t}$ on a product probability space). Then, the integrability properties of $\bar{M}_{t}+N_{t}^{t}$, and more generally of all random variables appearing below, come from those of $\bar{M}_{t}$ and $N_{t}^{t}$.

The function $\bar{q}(t, \cdot)$ will be the density of the random variable $\bar{Z}_{t}, t>0$.

It is readily seen that $p_{\bar{M}}(t, x)=t^{-d} p_{\bar{M}}\left(1, t^{-1} x\right), t>0, x \in \mathbb{R}^{d}$. Hence

$$
\bar{M}_{t} \stackrel{\text { (law) }}{=} t \bar{M}_{1}, \quad N_{t}^{t} \stackrel{\text { (law) }}{=} t N_{1}^{1} .
$$

By independence of $\bar{M}_{t}$ and $N_{t}^{t}$, using the Fourier transform, one can prove that

$$
\bar{Z}_{t} \stackrel{(\text { law })}{=} t \bar{Z}_{1} \text {. }
$$

Moreover, $\mathbb{E}\left[\left|\bar{Z}_{t}\right|^{\gamma}\right]=\mathbb{E}\left[\left|\bar{M}_{t}+N_{t}\right|^{\gamma}\right] \leq C_{\gamma} t^{\gamma}\left(\mathbb{E}\left[\left|\bar{M}_{1}\right|^{\gamma}\right]+\mathbb{E}\left[\left|N_{1}^{1}\right|^{\gamma}\right]\right) \leq C_{\gamma} t^{\gamma}, \gamma \in(0,1)$. This shows that the density of $\bar{Z}_{t}$ verifies (2.11).

The controls (2.10) on the derivatives are derived similarly using $(\mathrm{A.49})$ for all multi-index $\beta,|\beta| \leq 2$, and the same previous argument.

Now, the bounds of 2.13 involving diagonal perturbation again follow from the expression of $p_{\bar{M}}(t, \cdot)$ in A.49. Similar arguments apply to get 2.12. Also, still in 2.13), the bound on the fractional Laplacian is a consequence of the previous decomposition applying the operator $\Delta^{\frac{1}{2}}$ to $p_{M_{t}^{t}}(\cdot)$. Namely, it is easily checked that $\left|\Delta^{\frac{1}{2}} p_{M_{t}^{t}}(x)\right| \leq C t^{-1} p_{\bar{M}}(t, x)$ (see again Lemma 4.3 in [10] for details). Equations 2.15) and 2.14 eventually follow from the previous bounds introducing the diagonal/off-diagonal cut-off. Namely, for (2.15), if $\left|z-z^{\prime}\right|>t$, then $\left|D p_{Z_{t}}(z)-D p_{Z_{t}}\left(z^{\prime}\right)\right| \leq\left|D p_{Z_{t}}(z)\right|+\left|D p_{Z_{t}}\left(z^{\prime}\right)\right| \leq \frac{C}{t}\left(\frac{\left|z-z^{\prime}\right|}{t}\right)^{\beta}\left(\bar{q}(t, z)+\bar{q}\left(t, z^{\prime}\right)\right)$, whereas if $\left|z-z^{\prime}\right| \leq t$ then, from 2.10) $\left|D p_{Z_{t}}(z)-D p_{Z_{t}}\left(z^{\prime}\right)\right| \leq \int_{0}^{1} \bar{q}\left(t, z+\lambda\left(z^{\prime}-z\right)\right) \frac{\left|z-z^{\prime}\right|}{t^{2}} \leq \frac{C}{t} \bar{q}(t, z)\left(\frac{\left|z-z^{\prime}\right|}{t}\right)^{\beta}$, using 2.12 for the last inequality. The bound (2.14) can be derived in a similar way.

Lemma A.1. Let $b \in C_{b}^{2}\left(\mathbb{R}^{d}, \mathbb{R}^{d}\right)$ and assume that there exists $b_{0} \in \mathbb{R}^{d} \varepsilon \in(0,1)$, such that, for all $x \in \mathbb{R}^{d}$, $\left|b(x)-b_{0}\right| \leq \varepsilon\left(c f .\left(\mathbf{C}_{\varepsilon}\right)\right)$. Let us consider the pathwise unique solution $\left(X_{t}^{x}\right)$ to

$$
X_{t}=x+\int_{0}^{t} b\left(X_{s}\right) d s+Z_{t}, \quad t \geq 0
$$

(defined on a stochastic basis $\left(\Omega, \mathcal{F},\left(\mathcal{F}_{t}\right), \mathbb{P}\right)$ ) and the corresponding resolvent

$$
u_{\lambda}(x)=\mathbb{E}\left[\int_{0}^{+\infty} \exp (-\lambda s) f\left(X_{s}^{x}\right) d s\right], \lambda>0, x \in \mathbb{R}^{d} .
$$

Then, for $\lambda \geq 1$ and $p>d$, there exists $C=C\left(\varepsilon, d, p,\|b\|_{C_{b}^{2}}\right)$ such that

$$
\left|u_{\lambda}(x)\right| \leq C\|f\|_{L^{p}\left(\mathbb{R}^{d}\right)}, \quad f \in C_{0}^{\infty}\left(\mathbb{R}^{d}\right) .
$$

Proof. Thanks to the regularity of $b$ we know that $u=u_{\lambda}$ is the unique bounded classical solution to

$$
\lambda u(x)-L(x)-b(x) \cdot D u(x)=f(x), \quad x \in \mathbb{R}^{d},
$$

which we can write as $\lambda u(x)-L(x)-b_{0} \cdot D u(x)=f(x)+\left(b(x)-b_{0}\right) \cdot D u(x)$. It follows the representation formula

$$
u(x)=\int_{0}^{+\infty} \exp (-\lambda t) d t \int_{\mathbb{R}^{d}}\left[f(y)+\left(b(y)-b_{0}\right) \cdot D u(y)\right] p_{Z_{t}}\left(y-x-t b_{0}\right) d y .
$$

Now we use $\left|b(x)-b_{0}\right| \leq \varepsilon$ for $\varepsilon$ small enough and $(2.22)$. By a fixed point theorem in $W^{1, p}\left(\mathbb{R}^{d}\right), 1<p<\infty$, we find that $u \in W^{1, p}\left(\mathbb{R}^{d}\right)$ and, moreover,

$$
\|u\|_{W^{1, p}\left(\mathbb{R}^{d}\right)} \leq C\|f\|_{L^{p}\left(\mathbb{R}^{d}\right)} .
$$

Choosing $p>d$ and applying the Sobolev embedding theorem we obtain the assertion. 


\section{REFERENCES}

[1] R. F. Bass. Diffusions and Elliptic Operators Springer Science \& Business Media, 1998

[2] R. F. Bass. Stochastic differential equations driven by symmetric stable processes. Sminaire de probabilits de Strasbourg, Volume 36 (2002), p. 302-313

[3] R.F. Bass and Z.Q. Chen. Systems of equations driven by stable processes. Probab. Theory Related Fields, 134(2):175-214, 2006.

[4] P.-E. Chaudru de Raynal, S. Menozzi, and E. Priola. Schauder estimates for drifted fractional operators in the supercritical case. Preprint Arxiv 2019 published online in J. Funct. Anal. (https://doi.org/10.1016/j.jfa.2019.108425)

[5] Z.Q. Chen, L. Wang Uniqueness of stable processes with drift Proc. Amer. Math. Soc. 144 : 2661-2675, 2016

[6] R. Coifman and G. Weiss. Analyse Harmonique non-commutative sur certains espaces homogènes, volume 242. Springer, Lecture Notes in Math., 1971.

[7] E. Ethier and T. Kurtz Markov Processes. Characterization and Convergence. Wiley, 1986.

[8] W. Hoh. Pseudo-Differential Operators Generating Markov Processes. Habilitationsschrift. Universitat Bieleeld, Bielefeld 1998.

[9] L. Huang and S. Menozzi. A Parametrix Approach for some Degenerate Stable Driven SDEs. Annales Instit. H. Poincaré (B), 52:1925-1975, 2016.

[10] L. Huang, S. Menozzi, and E. Priola. $L^{p}$ Estimates For Degenerate Non-Local Kolmogorov Operators. Journal de Mathématiques Pures et Appliquées, 121:162-215, 2019.

[11] P. Jin. On weak solutions of SDEs with singular time-dependent drift and driven by stable processes Stochastics and Dynamics, $18: 1850013,2018$.

[12] T. Komatsu. Markov processes associated with certain integrodifferential operators Osaka J. Math. 10 : 271-303, 1973.

[13] T. Komatsu. On the martingale problem for generators of stable processes with perturbations. Osaka J. Math., 21 : 113-132, 1984.

[14] N. V. Krylov. Once more about the connection between elliptic operators and Itô's stochastic equations Statistics and control of stochastic processes (Moscow, 1984), 214-229, Transl. Ser. Math. Engrg., Optimization Software, New York, 1985.

[15] F. Kuhn. On Martingale Problems and Feller Processes. Eletron. J. Probab. 23 : 1-18, 2018.

[16] T. G. Kurtz. Equivalence of stochastic equation and martingale problem, Stochastic Analysis 2010 . D. Crisan (ed,), page 113-130. Springer-Verlag 2011

[17] R. Mikulevicius and H. Pragarauskas. On the Cauchy problem for integro-differential operators in Hölder classes and the uniqueness of the martingale problem. Potential Anal., 40(4):539-563, 2014.

[18] E. Priola. Pathwise uniqueness for singular SDEs driven by stable processes. Osaka J. Math., 49- 2:421-447, 2012.

[19] E. Priola, A. Shirikyan, L. Xu, J. Zabczyk. Exponential ergodicity and regularity for equations with Lévy noise Stochastic Processes and their Applications, 122 : 106-133, 2012.

[20] K. Sato. Lévy processes and Infinitely divisible Distributions. Cambridge University Press, 1999.

[21] D. W. Stroock and S.R.S Varadhan. Multidimensional diffusion processes. Grundlehren der Mathematischen Wissenschaften 233. Springer-Verlag, 1979.

[22] H. Tanaka, M. Tsuchiya, and S. Watanabe. Perturbation of drift-type for Lévy processes. J. Math. Kyoto Univ., 14:73-92, 1974.

[23] G. Zhao. Weak uniqueness for SDEs driven by supercritical stable processes with Holder drifts. Proc. Amer. Math. Soc. $147: 849-860,2019$.

Univ. Grenoble Alpes, Univ. Savoie Mont Blanc,CNRS, LAMA, 73000 Chambéry, France, Pe.Deraynal@univ-Smb.fr

Laboratoire de Moélisation Mathématique d’Evry, UMr CNRS 8071,, Université d’Evry Val D'Essonne, ParisSaclay, 23 Boulevard de France, 91037 Evry, France,, and, Laboratory of Stochastic Analysis, Higher School of Economics,, Pokrovsky Boulevard, 11, Moscow, Russian Federation, stephane.menozzi@univ-evry.Fr

Università di Pavia, Dipartimento di matematica, Via Adolfo Ferrata 5, 27100 Pavia. enrico.priola@Unipv.it 